\title{
METODO SEMI-EMPÍRICO PARA ESTIMAR LA RESISTENCIA LATERAL EN MUROS DE MAMPOSTERÍA CONFINADA
}

\author{
Sulpicio Sánchez Tizapa ${ }^{(1)}$, Ahmed Mebarki ${ }^{(1)}$
}

\begin{abstract}
RESUMEN
Se presenta un método semi-empírico para estimar la resistencia lateral en muros de mampostería confinada que propone la superficie de falla en la diagonal y considera la capacidad resistente de la mampostería y los castillos, de los cuales se requieren las características geométricas-mecánicas. La capacidad del método es mostrada con los resultados de 25 muros elaborados con bloques huecos de concreto, tabicones de jal-cemento y tabique rojo recocido, para los que se estimaron valores de resistencia lateral del $73 \%$ al $113 \%$ respecto de la resistencia experimental obtenida en ensayes bajo cargas laterales cíclicas y monótonas. La relación de forma $(\mathrm{H} / \mathrm{L})$ de los muros fue igual a $0.74,1.00$ y 1.26 y el esfuerzo vertical en los ensayes es representativo al actuante en edificios de hasta 5 niveles.
\end{abstract}

\begin{abstract}
A semi empiric method to evaluate the ultimate shear of confined masonry walls with the assumption of a failure surface by shear effect in the diagonal wall is presented. For its application, masonry and reinforced concrete properties are required. The model provides results that range within $73 \%$ up to $113 \%$ of the experimental strength of 25 walls tested under lateral cyclic and monotonic loads in Mexico and Venezuela, with several ratios H/L by using concrete hollow blocks and solid bricks. The applied vertical stresses are the usual values for buildings up to 5 levels.
\end{abstract}

\section{INTRODUCCIÓN}

Los muros de mampostería son construidos con piezas de arcilla o concreto pegados con mortero y confinados por castillos y dalas, estos elementos estructurales son ampliamente utilizados en Latinoamérica para la construcción de edificios y/o casas-habitación en zonas con solicitaciones importantes ante carga sísmica. Los tipos de falla comunes que presentan son: a) agrietamiento de las piezas o del mortero cuando el estado de esfuerzos actuante sobrepasa la resistencia en compresión o tensión, b) pérdida de adherencia en la unión pieza-mortero si el esfuerzo cortante excede el esfuerzo resistente (Andreus, 1996), (Meli y Salgado, 1969), (Sánchez, Mebarki, Soto y Arroyo, 2007) y, c) agrietamiento de los elementos confinantes por efecto cortante después de la falla de la mampostería (Flores y Alcocer, 2001), (Hernández y Urzúa, 2002).

Para evaluar el comportamiento de los muros de mampostería confinada hay dos opciones, la primera basada en la modelación con elementos finitos mediante macro o micro modelación, que requiere un proceso de homogenización o un alto esfuerzo computacional (Abdou, 2005) considerando el

Artículo recibido el 27 de octubre de 2006 y aprobado para su publicación el 4 de mayo de 2009. Se aceptarán comentarios y/o discusiones hasta cinco meses después de su publicación

(1) Université Paris-Est, Laboratoire Modélisation et Simulation Multi Echelle, MSME FRE3160 CNRS, 5 bd Descartes, 77454 Marne-La-Vallée, France. Email: sstizapa@hotmail.com, Ahmed.Mebarki@univ-mlv.fr 
comportamiento no lineal desde bajos niveles de esfuerzos. En la segunda opción están los métodos simplificados donde la mampostería es sustituida por una diagonal equivalente con falla en compresión o por una diagonal sujeta a tensión diagonal (Cruz y Mebarki, 2002), (Sánchez y Mebarki, 2006), (Mebarki Et al., 2009). Una desventaja del método de la diagonal en compresión es la evaluación del ancho equivalente, sin significado físico, mientras que para el método de falla por tensión diagonal existe indefinición respecto a la aportación del esfuerzo vertical.

Para evitar los problemas antes mencionados, los autores proponen un método semi-empírico basado en criterios de equilibrio y comportamiento de cuerpo rígido, que supone un modo de falla de agrietamiento en la diagonal cuando el esfuerzo cortante en el sistema pieza-mortero excede el esfuerzo cortante de la mampostería (NTCM, 2000a), (ASTM, 2007). Este método considera también, la influencia del esfuerzo vertical actuante y la capacidad cortante de los castillos.

\section{INFORMACIÓN EXPERIMENTAL}

Los resultados analíticos fueron comparados con los resultados obtenidos en nueve muros ensayados en Venezuela y 16 en México. Las características generales y el tipo de carga aplicada en los ensayes son presentados en la tabla 1, donde $t$ es el espesor, $H$ la altura y $L$ la longitud. La notación entre paréntesis de la primera columna corresponde a la utilizada en las referencias. En las series de muros 1(1)9(9) construidos por Castilla y Marinilli (2003) y 10(421)-17(604) por Treviño et al. (2004) fueron utilizados bloques huecos de concreto y el lote de muros 18(MD1)-20(MD3) fue elaborado con tabicones de jal-cemento por Hernández y Urzúa (2002). Finalmente, los muros 21(M2)-25(804) fueron construidos con tabique rojo recocido, el muro 21(M2) fue ensayado por Aguilar y Alcocer (1997) y los muros 22(801)-25(804) por Meli y Salgado (1969). La tabla 2 muestra la cuantía de acero longitudinal, $\rho$, la dimensión $h_{c}$ de los castillos paralela al plano del muro y el peralte total de la dala superior, $h_{d}$, el espesor de estos elementos fue igual al espesor del muro.

Las propiedades mecánicas requeridas son presentadas en la tabla 3 donde $v_{m}^{*}$ es la resistencia de diseño, $\sigma$ es el esfuerzo vertical aplicado, $f_{m}^{*}$ representa la resistencia a compresión de la mampostería, $f_{c}^{\prime}$ es la resistencia a compresión del concreto, $E_{c}$ es el módulo elástico del concreto y $E_{m}$ es el módulo elástico de la mampostería. Para los muros ensayados en México sin información de los módulos elásticos se utilizaron las ecuaciones de la norma (NTCM, 2004a), (NTCC, 2004b). En los muros 1(1)-9(9) la resistencia de diseño $v_{m}{ }_{m}$ se obtuvo mediante la ecuación 1 con la resistencia media $\bar{v}_{m}$ reportada por Castilla y Marinilli (2003) y un coeficiente de variación propuesto $c_{v}=0.2$, los módulos elásticos fueron evaluados con las ecuaciones 2 y 3 (MINDUR, 1987). Respecto a la carga experimental $V_{\text {exp }}$ de los muros ensayados ante carga cíclica, el valor registrado fue el promedio de los valores máximos en cada dirección

$$
\begin{aligned}
& v_{m}^{*}=\overline{v_{m}} /\left(1+2.5 c_{v}\right) \\
& E_{c}=15100 \sqrt{f_{c}^{\prime}} \\
& E_{m}=800 f_{m}^{*}
\end{aligned}
$$


Tabla 1. Características generales y tipo de carga aplicada

\begin{tabular}{ccccccc}
\hline Muro & $\begin{array}{c}L, \\
(\mathrm{~cm})\end{array}$ & $\begin{array}{c}H, \\
(\mathrm{~cm})\end{array}$ & $H / L$ & $\begin{array}{c}t \\
(\mathrm{~cm})\end{array}$ & $\begin{array}{c}\text { Tipo de } \\
\text { carga }\end{array}$ & Tipo de pieza \\
\hline $1(1)$ & 236 & 230 & 0.97 & 15 & Cíclica & Bloque hueco de concreto \\
$2(2)$ & 236 & 230 & 0.74 & 15 & Cíclica & Bloque hueco de concreto \\
$3(3)$ & 236 & 230 & 1.27 & 15 & Cíclica & Bloque hueco de concreto \\
$4(4)$ & 312 & 230 & 1.27 & 15 & Cíclica & Bloque hueco de concreto \\
$5(5)$ & 312 & 230 & 1.27 & 15 & Cíclica & Bloque hueco de concreto \\
$6(6)$ & 312 & 230 & 1.27 & 15 & Cíclica & Bloque hueco de concreto \\
$7(7)$ & 182 & 230 & 1.27 & 15 & Cíclica & Bloque hueco de concreto \\
$8(8)$ & 182 & 230 & 1.27 & 15 & Cíclica & Bloque hueco de concreto \\
(9) & 182 & 230 & 1.27 & 15 & Cíclica & Bloque hueco de concreto \\
10(421) & 250 & 245 & 0.98 & 14 & Cíclica & Bloque hueco de concreto \\
11(422) & 250 & 245 & 0.98 & 14 & Cíclica & Bloque hueco de concreto \\
12(423) & 250 & 245 & 0.98 & 14 & Cíclica & Bloque hueco de concreto \\
13(424) & 250 & 245 & 0.98 & 14 & Cíclica & Bloque hueco de concreto \\
14(601) & 250 & 245 & 0.98 & 14 & Cíclica & Bloque hueco de concreto \\
15(602) & 250 & 245 & 0.98 & 14 & Cíclica & Bloque hueco de concreto \\
16(603) & 250 & 245 & 0.98 & 14 & Cíclica & Bloque hueco de concreto \\
17(604) & 250 & 245 & 0.98 & 14 & Cíclica & Bloque hueco de concreto \\
18(MD1) & 250 & 250 & 1.00 & 14 & Cíclica & Tabicón jal-cemento \\
19(MD2) & 250 & 250 & 1.00 & 14 & Cíclica & Tabicón jal-cemento \\
20(MD3) & 250 & 250 & 1.00 & 14 & Cíclica & Tabicón jal-cemento \\
21(M2) & 250 & 250 & 1.00 & 13 & Cíclica & Tabique de barro recocido \\
22(801) & 200 & 200 & 1.00 & 12 & Monótona & Tabique de barro recocido \\
23(802) & 200 & 200 & 1.00 & 12 & Monótona & Tabique de barro recocido \\
24(803) & 200 & 200 & 1.00 & 12 & Monótona & Tabique de barro recocido \\
25(804) & 200 & 200 & 1.00 & 12 & Monótona & Tabique de barro recocido \\
\hline & & & & & &
\end{tabular}

Tabla 2. Dimensiones de los elementos confinantes y cuantía de refuerzo longitudinal

\begin{tabular}{|c|c|c|c|c|c|c|c|}
\hline \multirow{2}{*}{ Muro } & Castillo & Dala & \multirow{2}{*}{$p$} & \multirow{2}{*}{ Muro } & Castillo & Dala & \multirow{2}{*}{$p$} \\
\hline & $h_{c}, \mathrm{~cm}$. & $h_{d}, \mathrm{~cm}$. & & & $h_{c}, \mathrm{~cm}$. & $h_{d}, \mathrm{~cm}$. & \\
\hline $1(1)$ & 15 & 20 & 0.031 & 14(601) & 15 & 15 & 0.005 \\
\hline $2(2)$ & 15 & 20 & 0.031 & $15(602)$ & 15 & 15 & 0.005 \\
\hline $3(3)$ & 15 & 20 & 0.031 & 16(603) & 15 & 15 & 0.005 \\
\hline 4(4) & 15 & 20 & 0.031 & 17(604) & 15 & 15 & 0.005 \\
\hline $5(5)$ & 15 & 20 & 0.031 & 18(MD1) & 15 & 10 & 0.013 \\
\hline $6(6)$ & 15 & 20 & 0.031 & 19(MD2) & 15 & 10 & 0.013 \\
\hline $7(7)$ & 15 & 20 & 0.031 & 20(MD3) & 15 & 10 & 0.013 \\
\hline $8(8)$ & 15 & 20 & 0.031 & 21(M2) & 15 & 10 & 0.013 \\
\hline $9(9)$ & 15 & 20 & 0.031 & 22(801) & 20 & 20 & 0.023 \\
\hline $10(421)$ & 15 & 15 & 0.013 & 23(802) & 20 & 20 & 0.035 \\
\hline $11(422)$ & 15 & 15 & 0.013 & 24(803) & 20 & 20 & 0.035 \\
\hline 12(423) & 15 & 15 & 0.013 & $25(804)$ & 20 & 20 & 0.035 \\
\hline $13(424)$ & 15 & 15 & 0.013 & & & & \\
\hline
\end{tabular}


Tabla 3. Propiedades mecánicas y resistencia última

\begin{tabular}{cccccccc}
\hline Muro & $\begin{array}{c}v^{*}{ }_{m}, \\
\mathrm{~kg} / \mathrm{cm}^{2}\end{array}$ & $\begin{array}{c}\sigma, \\
\mathrm{kg} / \mathrm{cm}^{2}\end{array}$ & $\begin{array}{c}f_{m}^{*}, \\
\mathrm{~kg} / \mathrm{cm}^{2}\end{array}$ & $\begin{array}{c}f_{c}^{\prime}, \\
\mathrm{kg} / \mathrm{cm}^{2}\end{array}$ & $\begin{array}{c}E_{c}, \\
\mathrm{~kg} / \mathrm{cm}^{2}\end{array}$ & $\begin{array}{c}E_{m}, \\
\mathrm{~kg} / \mathrm{cm}^{2}\end{array}$ & $\begin{array}{c}V_{\text {exp }}, \\
\text { ton. }\end{array}$ \\
\hline $1(1)$ & 5.0 & 8.0 & 80.0 & 300.0 & 261540 & 64000 & 28.0 \\
$2(2)$ & 5.0 & 4.0 & 80.0 & 300.0 & 261540 & 64000 & 23.8 \\
$3(3)$ & 5.0 & 0.0 & 80.0 & 300.0 & 261540 & 64000 & 18.6 \\
$4(4)$ & 5.0 & 6.0 & 80.0 & 300.0 & 261540 & 64000 & 38.0 \\
$5(5)$ & 5.0 & 0.0 & 80.0 & 300.0 & 261540 & 64000 & 13.7 \\
$6(6)$ & 5.0 & 4.0 & 80.0 & 300.0 & 261540 & 64000 & 31.7 \\
$7(7)$ & 5.0 & 8.0 & 80.0 & 300.0 & 261540 & 64000 & 21.0 \\
8(8) & 5.0 & 0.0 & 80.0 & 300.0 & 261540 & 64000 & 16.1 \\
9(9) & 5.0 & 4.0 & 80.0 & 300.0 & 261540 & 64000 & 17.3 \\
$10(421)$ & 2.9 & 5.5 & 38.9 & 235.0 & 122638 & 40333 & 15.7 \\
11(422) & 2.9 & 5.5 & 38.9 & 235.0 & 122638 & 40333 & 16.0 \\
$12(423)$ & 2.9 & 5.5 & 38.9 & 235.0 & 122638 & 40333 & 19.3 \\
13(424) & 2.9 & 5.5 & 38.9 & 235.0 & 122638 & 40333 & 17.7 \\
14(601) & 2.9 & 5.5 & 38.9 & 235.0 & 122638 & 40333 & 16.6 \\
15(602) & 2.9 & 5.5 & 38.9 & 235.0 & 122638 & 40333 & 17.0 \\
$16(603)$ & 2.9 & 5.5 & 38.9 & 235.0 & 122638 & 40333 & 17.1 \\
17(604) & 2.9 & 5.5 & 38.9 & 235.0 & 122638 & 40333 & 16.2 \\
18(MD1) & 2.1 & 3.7 & 15.0 & 180.0 & 107331 & 19422 & 14.0 \\
19(MD2) & 1.9 & 3.7 & 26.0 & 180.0 & 107331 & 20353 & 14.4 \\
20(MD3) & 3.5 & 3.7 & 20.7 & 180.0 & 107331 & 23704 & 14.3 \\
21(M2) & 2.6 & 5.0 & 27.0 & 280.0 & 111090 & 7285 & 14.1 \\
22(801) & 3.5 & 0.0 & 102.0 & 104.0 & 81584 & 11000 & 5.8 \\
23(802) & 3.5 & 2.9 & 102.0 & 159.0 & 100876 & 11000 & 13.5 \\
24(803) & 3.5 & 0.0 & 102.0 & 237.0 & 123158 & 11000 & 11.0 \\
25(804) & 8.1 & 0.0 & 102.0 & 331.0 & 254708 & 11000 & 19.5 \\
\hline
\end{tabular}

\section{MÉTODO PROPUESTO}

\section{Comportamiento de muros de mampostería confinada}

A continuación se describe el comportamiento de los muros referenciados sobre el cual se fundamenta este método. Aguilar y Alcocer (1997) reportan el primer agrietamiento del muro 21(M2) en el ciclo $+3(R=0.13 \%)$ y la presencia de una grieta principal en la diagonal para el ciclo $+5(R=0.46 \%)$ cuando se alcanza la resistencia máxima, después el castillo se agrieta por efecto cortante en el ciclo +6 . Aquí, la variable $R$ representa la distorsión expresada por la relación desplazamiento lateral/altura del muro. La figura 1 presenta la curva fuerza cortante vs distorsión donde el último punto corresponde a la primera plastificación del acero longitudinal después del agrietamiento del concreto y la mampostería. Al final del ensaye las piezas presentaron agrietamiento y en algunas zonas se agrietaron también las juntas verticales y horizontales mientras que los castillos tuvieron grietas a $45^{\circ}$ respecto de la horizontal.

La aparición del primer agrietamiento inclinado en muros con piezas huecas (Treviño Et al., 2004) fue simultáneo a pequeñas fisuras por flexión en los castillos con una distorsión $R=0.10 \%$. Posteriormente las grietas en las piezas aumentaron de longitud y tamaño y aparecieron otras, al final ocurrió el desprendimiento generalizado de las paredes de las piezas ubicadas en la diagonal y los extremos 
inferiores de los castillos sufrieron poco deterioro. Sin embargo, se apreciaron diferencias en el grado de daño a favor de los muros con estribos concentrados en los extremos, adicionalmente se observó el agrietamiento de los castillos en sus esquinas superiores, ver figura 2.

Hernández y Urzúa (2002) ensayaron tres muros construidos con piezas macizas, en el muro 18(MD1) el daño fue concentrado en dos grietas inclinadas que penetraron en los extremos de ambos castillos con un aplastamiento severo de las piezas en la intersección de las diagonales. Al final del ensaye del muro 19(MD2) se registró un patrón definido de grietas con una inclinación ligeramente mayor a $45^{\circ}$ y daño semejante al presentado en el muro 18(MD1), para ambos casos, las grietas penetraron en los castillos. La figura 1 presenta la envolvente positiva de los tres muros ensayados.

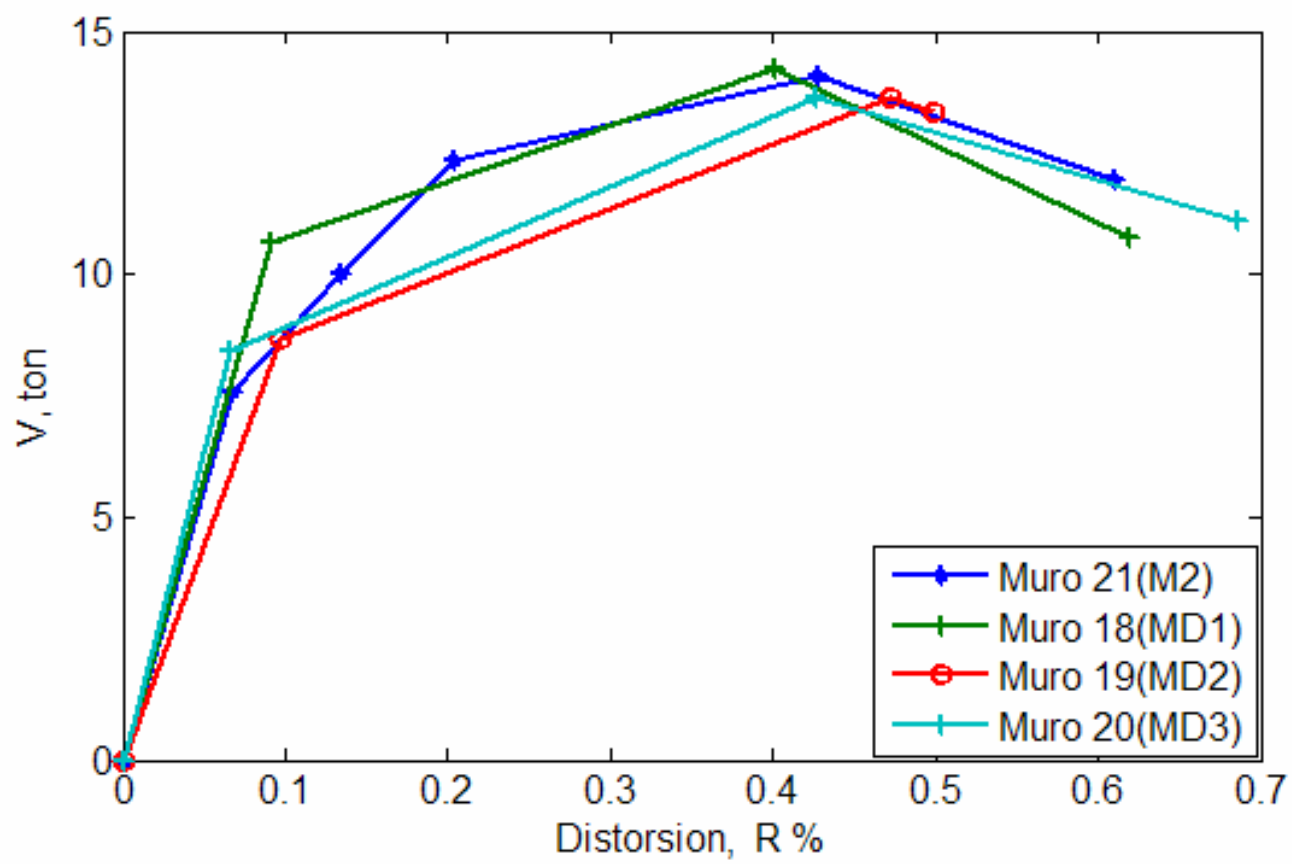

Figura 1. Envolvente de resistencia vs. distorsión en ciclos positivos (Hernández y Urzua, 2002; Aguilar y Alcocer, 1997)

Meli y Salgado (1969) ensayaron muros ante carga monótona y observaron que la carga máxima no corresponde a la aparición de la grieta diagonal, ésta se desarrolla lentamente del centro hacia los extremos y cruza los castillos cuando se tiene la carga máxima resistente. También observaron que la diferencia entre la carga de agrietamiento y la carga máxima depende de la resistencia del marco exterior.

Flores y Alcocer (2001) detectaron un comportamiento elástico-lineal en los primeros ciclos cuando la distorsión era menor a la del primer agrietamiento diagonal, después comenzó la degradación de rigidez al extenderse el agrietamiento sobre la diagonal y el muro quedó formado por dos cuñas triangulares de mampostería confinadas aún por el marco de concreto. Finalmente, la resistencia máxima fue registrada cuando las grietas penetraron en los castillos. El patrón de daño es mostrado en la figura 3 y la envolvente de carga trilineal fuerza cortante vs. distorsión fue dibujada en la figura 4, donde el punto $\boldsymbol{a}$ corresponde al primer agrietamiento diagonal de la mampostería, el punto $\boldsymbol{b}$ está asociado a la resistencia máxima y el punto $\boldsymbol{c}$ corresponde a la fluencia del acero longitudinal. 


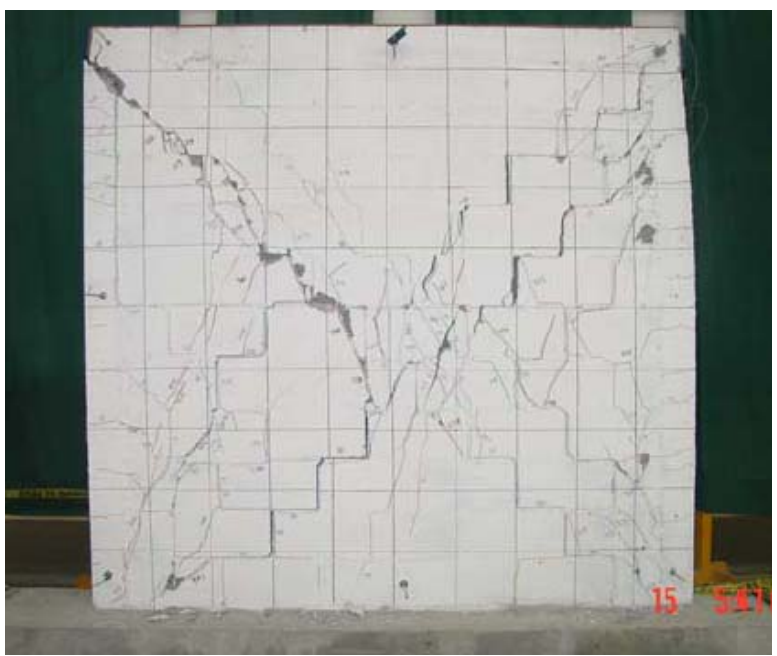

Figura 2. Estado final del muro 11(422) (Treviño Et al. 2004)
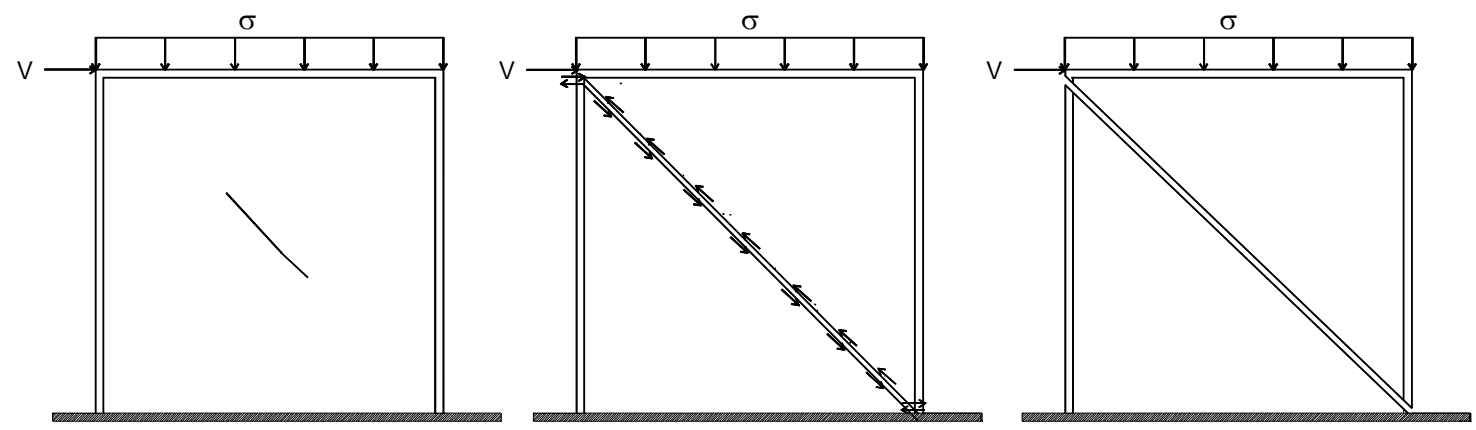

Primer agrietamiento en el punto $\boldsymbol{a}$

Estado intermedio Resistencia máxima en el punto $\boldsymbol{b}$ Figura 3. Estado de daño con distintos niveles de distorsión (Flores y Alcocer, 2001)

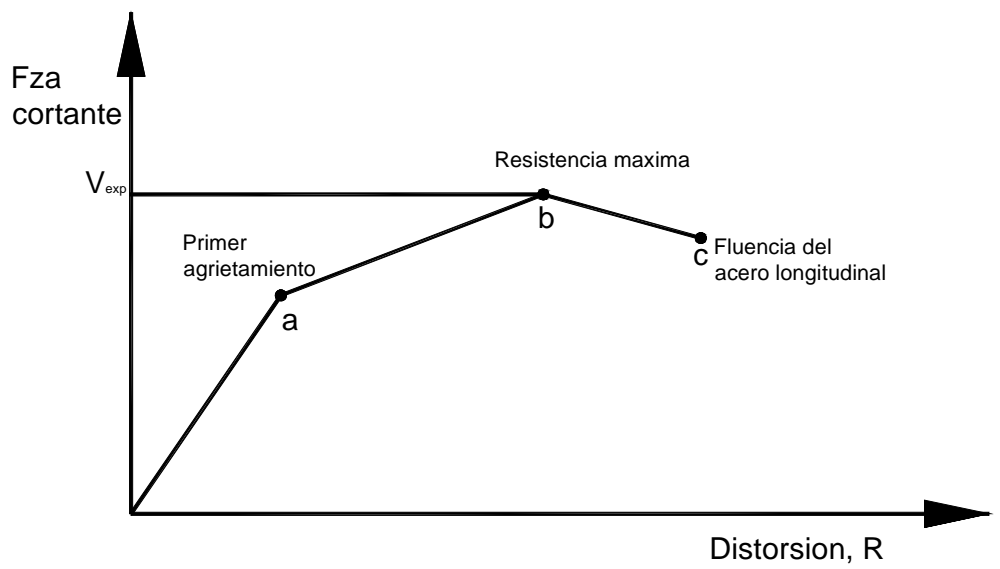

Figura 4. Estados de daño y envolvente de resistencia vs. distorsión (Flores y Alcocer, 2001) 
En general, los muros sujetos a carga cíclica tuvieron idéntica resistencia en las dos direcciones y los desplazamientos de cortante fueron mayores que los de flexión. Adicionalmente, se observó un aumento de resistencia ante la presencia de esfuerzo vertical que en algunos casos alcanzó el esfuerzo de fluencia.

\section{Cálculo de la resistencia máxima}

El método semi-empírico propuesto está basado en las siguientes hipótesis:

1. La falla del muro es generada por el agrietamiento simultáneo de la diagonal causado por efecto cortante.

2. El muro o cualquiera de sus dos partes tiene un comportamiento de cuerpo rígido antes y después de la falla respecto a su diagonal.

3. Las dalas superior e inferior tienen rigidez infinita ante flexión y ante carga axial.

La figura 5 presenta las fuerzas actuantes en un muro de mampostería donde $V$ es la fuerza horizontal inducida por sismo, $\sigma$ es el esfuerzo vertical aplicado, $L$ es la longitud, $H$ es la altura y $\gamma$ es el ángulo de la diagonal respecto a la vertical. El diagrama de cuerpo libre del nodo B muestra la fuerza horizontal $V$ induciendo tensión en el castillo y compresión en la diagonal que genera un esfuerzo cortante en la mampostería.
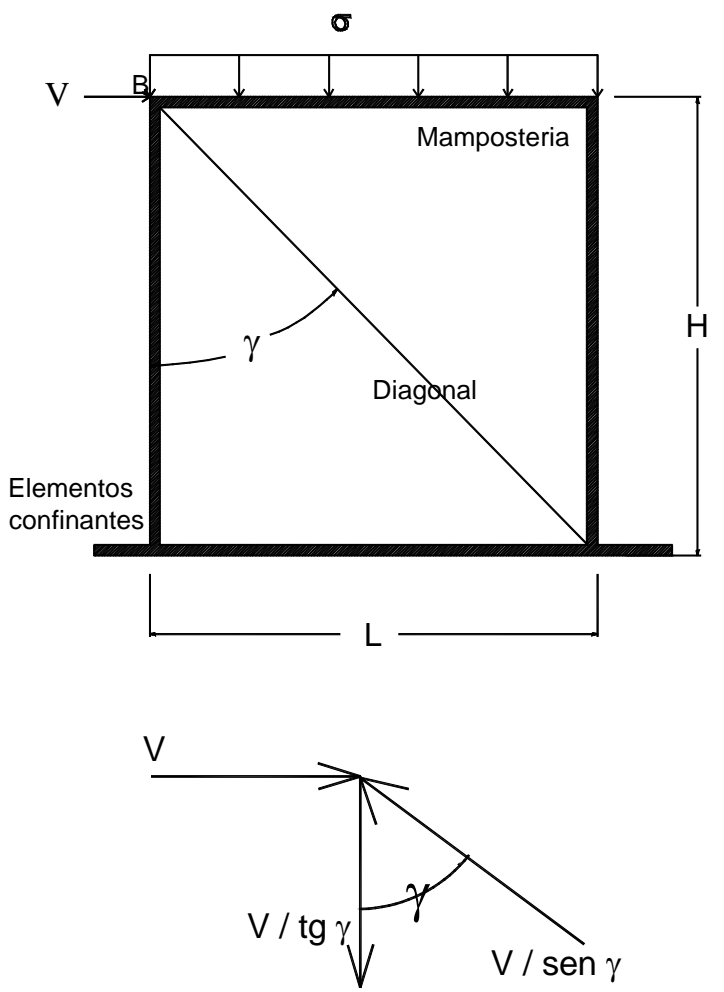

Figura 5. Muro de mampostería confinada sujeto a cargas y diagrama de cuerpo libre del nodo B

El objetivo del método es conocer la fuerza máxima asociada al punto $\boldsymbol{b}$ de la figura 4 que representa la resistencia del muro y es obtenida con las ecuaciones presentadas a continuación. 
a) Resistencia de la mampostería. La capacidad de un murete ensayado a compresión diagonal es la carga de colapso $P$ con la que se evalúa la resistencia media $v_{m}$ según la ecuación 4 (NTCM, 2004a), en un proceso inverso se puede calcular $P$ cuya dirección coincide con la diagonal del murete mediante la ecuación 5. Por otro lado, si se acepta que el muro mostrado en la figura 6 es un ensamble de muretes cuya diagonal coincide con las diagonales de los $N$ muretes sujetos a compresión diagonal, la resistencia total $P_{m}$ es la sumatoria de la resistencia $P_{i}$ de los $N$ muretes calculada con la ecuación 6 . En esta ecuación la resistencia media $v_{m}$ es sustituida por el término $v_{m}{ }_{m}$ que considera el efecto de flexión y el factor de tamaño del muro. En las ecuaciones 4, 5 y 6, $A_{d}$ es el área de la diagonal del murete y $A_{d m}$ es el área de la mampostería en la diagonal del muro delimitada por los castillos.

$v_{m}=P / A_{d}$

$P=v_{m} A_{d}$

$P_{m}=\sum_{i=1}^{N} v_{m}^{*} A_{d m i}=v_{m}^{*} \sum_{i=1}^{N} A_{d m i}=v_{m}^{*} A_{d m}$

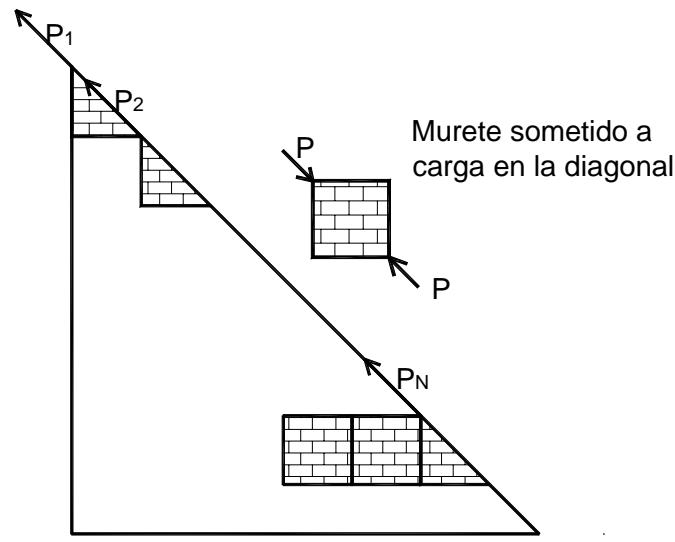

Figura 6. Resistencia de la mampostería

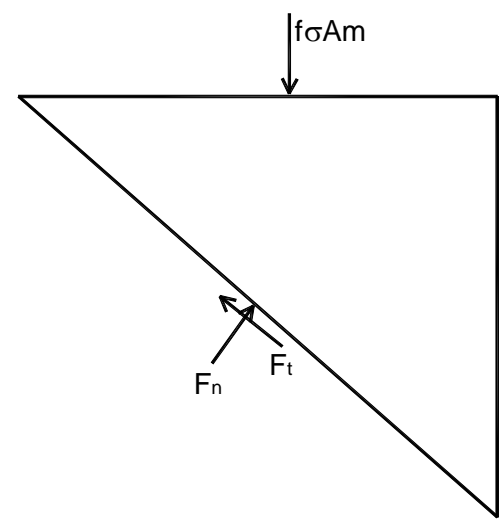

Figura 7. Efecto de la carga vertical

b) Efecto de la carga axial. En la parte superior de la mampostería actúa la fuerza vertical $f \sigma A_{m}$, equilibrada en el plano de falla por la fuerza tangencial $F_{t}$ y la fuerza normal $F_{n}$, el parámetro $f$ relaciona la rigidez vertical de la mampostería respecto a la rigidez vertical del muro y se obtiene con la ecuación 7. La fuerza tangencial $F_{t}$ es calculada con la ecuación 8 obtenida de la condición de equilibrio aplicada a la cuña superior del muro como lo muestra la figura 7. Las variables son: $A_{m}$ área horizontal de la mampostería, $A_{c}$ área de los castillos, $E_{c}$ y $E_{m}$ los módulos elásticos del concreto y mampostería, respectivamente y $\gamma$ es el ángulo de la diagonal del muro respecto a la vertical

$$
f=A_{m} E_{m} /\left(2 A_{c} E_{c}+A_{m} E_{m}\right)
$$




$$
F_{t}=f \sigma A_{d m} \operatorname{sen} \gamma \cos \gamma
$$

La primera hipótesis del método permite superponer la fuerza causada por el esfuerzo vertical $F_{t} \mathrm{y}$ la resistencia de la mampostería $P_{m}$. La fuerza resistente en la diagonal se expresa entonces por la ecuación 9 y la proyección horizontal, que es la resistencia de la mampostería, es calculada con la ecuación 10.

$F_{t t}=A_{d m}\left(v_{m}^{*}+f \sigma \operatorname{sen} \gamma \cos \gamma\right)$

$V_{m}=A_{d m} \operatorname{sen} \gamma\left(v_{m}^{*}+f \sigma \operatorname{sen} \gamma \cos \gamma\right)$

c) El agrietamiento de cortante en los castillos es común en la mayoría de los muros estudiados. Por lo anterior, la resistencia a cortante del concreto es agregada y calculada con las ecuaciones 11 o 12 (NTCC, 2004b) donde $t$ y $d$ son ancho y peralte efectivo, respectivamente, $\rho$ es la cuantía de acero longitudinal y $f_{c}^{*}$ es la resistencia reducida a compresión.

Si $\rho<0.015$

$V_{c r}=(0.20+20 \rho) t d \sqrt{f_{c}^{*}}$

Si $\rho>=0.015$

$V_{c r}=0.50 t d \sqrt{f_{c}^{*}}$

Finalmente, la ecuación 13 evalúa la capacidad del muro expresada como la suma de la resistencia de la mampostería y de la sección de concreto en los dos castillos. La ecuación 14 (NTCM, 2004a) utilizada para evaluar la resistencia en el primer agrietamiento diagonal y la ecuación 15 propuesta por Castilla y Marinilli (2003) para los muros 1(1)-9(9) son también presentadas, donde $A_{T}$ es la superficie

horizontal del muro. La ecuación 16 expresa la relación de resistencia calculada del criterio $i\left(V_{i}\right)$ respecto a la resistencia experimental $\left(V_{\text {exp }}\right)$.

$$
\begin{aligned}
& V_{C A L}=V_{m}+2 V_{c r} \\
& V_{\text {NTCM }}=A_{T}\left(0.50 v_{m}^{*}+0.30 \sigma\right) \leq 1.5 v_{m}^{*} A_{T} \\
& V_{V E N}=A_{T}\left(22.3 \sigma / f_{m}^{*}+5.0\right) \\
& k=V_{i} / V_{\exp }
\end{aligned}
$$

\section{RESULTADOS Y COMENTARIOS}

Existen dos muros donde la resistencia calculada varía significativamente respecto a la experimental, uno de ellos es el muro 22(801) que presentó el agrietamiento súbito del castillo en tensión 
e impidió el aumento de carga (Meli y Salgado, 1969), para el muro 5(5) no existe la descripción del colapso. La tabla 4 presenta la razón de resistencia calculada vs. resistencia experimental de los 25 muros con los diferentes criterios. El método propuesto predice una aportación de la mampostería igual al 85 \% de la resistencia y $14 \%$ para los castillos, la suma de ambos valores es el valor medio $k=0.98$ con un coeficiente de variación de 0.21 . El valor mínimo registrado fue $k=0.73$ en el muro 23(802) de tabique rojo recocido y el máximo valor, $k=1.70$, en el muro 5(5) de bloque hueco de cemento. Respecto a la resistencia al primer agrietamiento se obtuvo un valor medio $k=0.59$ según la ecuación 14 (NTCM, 2004a) mientras que la ecuación 15 (Castilla y Marinilli, 2003) proporcionó un valor medio $k=1.44$. La figura 8 presenta el histograma de $V_{C A L} / V_{\text {exp }}$ donde se observa que el $92 \%$ de resultados se encuentran entre los límites de $k=0.70$ y $k=1.2$ con dos valores extremos correspondientes a los muros 5(5) y 22(801). La ecuación 14 es utilizada en México para evaluar la resistencia de diseño, bajo esta idea se puede considerar que las construcciones tienen una sobreresistencia igual a 1.7 considerando un factor unitario de reducción de resistencia.

Tabla 4. Parámetros estadísticos para la totalidad de muros

\begin{tabular}{ccccc}
\hline Muro & $\mathrm{V}_{\mathrm{m}} / \mathrm{V}_{\exp }$ & $\mathrm{V}_{\mathrm{CAL}} / \mathrm{V}_{\exp }$ & $\mathrm{V}_{\mathrm{NTCM}} / \mathrm{V}_{\exp }$ & $\mathrm{V}_{\mathrm{VEN}} / \mathrm{V}_{\exp }$ \\
\hline Media & $\mathbf{0 . 8 5}$ & $\mathbf{0 . 9 8}$ & $\mathbf{0 . 5 9}$ & $\mathbf{1 . 4 4}$ \\
D estándar & 0.19 & 0.21 & 0.10 & 0.53 \\
C variación & 0.23 & 0.21 & 0.17 & 0.37 \\
\hline
\end{tabular}

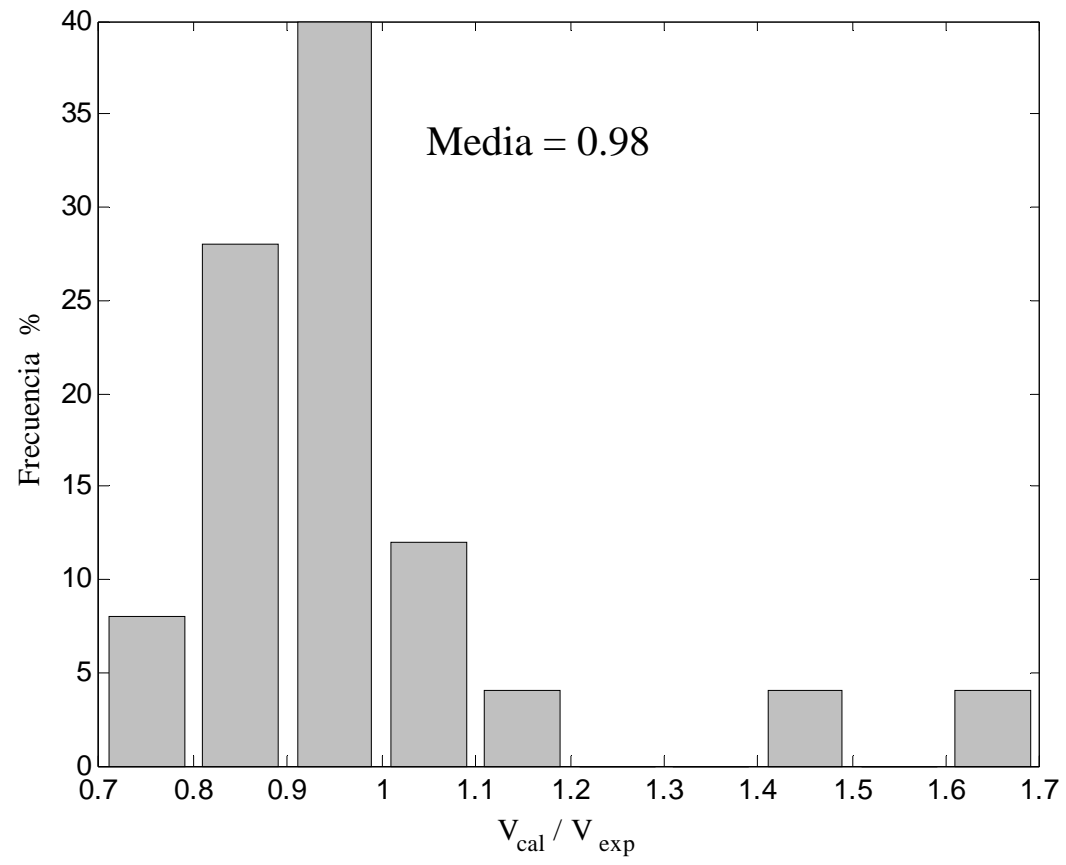

Figura 8. Histograma de la relación resistencia calculada (ecuación 13) vs. resistencia experimental

Los resultados en muros elaborados con tabicón jal-cemento son presentados en la tabla 5 con un valor medio $k=0.91$ para el método propuesto y $k=0.58$ según la ecuación 14 . Para estos muros la mampostería aporta $78 \%$ de la resistencia y los castillos aportan $13 \%$. En muros de tabique rojo recocido 
la participación de la mampostería disminuye al $76 \%$ y en los castillos aumenta al 22\% como se muestra en la tabla 6 , siendo el valor medio $k=0.98$.

Tabla 5. Relación resistencia calculada vs. resistencia experimental

\begin{tabular}{ccccc}
\multicolumn{5}{c}{ y parámetros estadísticos en muros de tabicón jal-cemento } \\
\hline Muro & $V_{m} / V_{\text {exp }}$ & $V_{\text {CAL }} / V_{\text {exp }}$ & $V_{N T C M} / V_{\text {exp }}$ & $V_{V E N} / V_{\text {exp }}$ \\
\hline 18(MD1) & 0.69 & 0.83 & 0.54 & 2.63 \\
19(MD2) & 0.64 & 0.77 & 0.50 & 1.99 \\
20(MD3) & 1.00 & 1.13 & 0.70 & 2.20 \\
Media & $\mathbf{0 . 7 8}$ & $\mathbf{0 . 9 1}$ & $\mathbf{0 . 5 8}$ & 2.27 \\
D estándar & 0.20 & 0.20 & 0.11 & 0.33 \\
C variación & 0.25 & 0.22 & 0.18 & 0.14 \\
\hline
\end{tabular}

Tabla 6. Relación resistencia calculada vs resistencia experimental y parámetros estadísticos en muros de tabique rojo recocido

\begin{tabular}{ccccc}
\hline Muro & $V_{m} / V_{\text {exp }}$ & $V_{C A L} / V_{\text {exp }}$ & $V_{N T C M} / V_{\text {exp }}$ & $V_{V E N} / V_{\text {exp }}$ \\
\hline 21(M2) & 0.69 & 0.84 & 0.65 & 2.10 \\
$22(801)$ & 1.16 & 1.48 & 0.72 & 2.07 \\
$23(802)$ & 0.56 & 0.73 & 0.47 & 1.00 \\
$24(803)$ & 0.61 & 0.87 & 0.38 & 1.09 \\
25(804) & 0.80 & 0.97 & 0.50 & 0.62 \\
Media & $\mathbf{0 . 7 6}$ & $\mathbf{0 . 9 8}$ & $\mathbf{0 . 5 4}$ & $\mathbf{1 . 3 8}$ \\
D estándar & 0.24 & 0.29 & 0.14 & 0.67 \\
C variación & 0.31 & 0.30 & 0.26 & 0.49 \\
\hline
\end{tabular}

Los resultados en los muros elaborados con bloques huecos son presentados en la tabla 7 que muestra $88 \%$ de resistencia aportada por la mampostería y $12 \%$ de resistencia aportada por los castillos con un valor medio $k=1.00 \mathrm{y}$ el coeficiente de variación igual a 0.20 . Las ecuaciones 14 y 15 proporcionan valores medios de $k=0.61$ y $k=1.32$, respectivamente. En esta tabla se observa la excelente aproximación de la ecuación 15 en los muros 1(1)-9(9).

Es posible obtener una ecuación semejante a la ecuación 14 (NTCM, 2004a) mediante la sustitución de las ecuaciones 17 y 18 en la ecuación 10 para obtener la ecuación 19, donde $A_{m}$ es el área horizontal de la mampostería y $F_{v}$ es función de la rigidez axial mampostería/concreto y de la relación de forma $H / L$. Es interesente observar que el valor de $F_{v}$ para los dos primeros tipos de piezas es próximo al expresado en la norma (NTCM, 2004a) según la tabla 8 pero difiere en el caso de los muros de tabique rojo recocido.

$$
\begin{aligned}
& A_{m}=A_{d m} \operatorname{sen} \gamma \\
& F_{v}=f \operatorname{sen} \gamma \cos \gamma \\
& V_{m}=A_{m}\left(v_{m}^{*}+F_{v} \sigma\right)
\end{aligned}
$$


La aplicación del método en muros con castillos intermedios donde no se garantiza el agrietamiento simultáneo de las diagonales podría sobrestimar la aportación de los castillos; desgraciadamente, no se encontró información experimental para aplicarlo y comprobar los resultados. La única referencia de una estructura ensayada a escala natural con varios muros es citada por Flores y Alcocer (2001) y se trata de una construcción de dos niveles, denominada 3D, con dos sistemas de muros paralelos por nivel y una separación entre ellos de $2.5 \mathrm{~m}$, cada sistema se integra por dos muros separados con un hueco intermedio. El objetivo fue identificar la influencia de los 4 castillos en cada lado y los resultados están en la tabla 9 que indican una razón $V_{C A L} / V_{\text {exp }}=1.14$, el valor de $V_{\text {exp }}$ fue calculado como el promedio del cortante obtenido en cada dirección de carga.

Tabla 7. Relación Resistencia calculada vs resistencia experimental y parámetros estadísticos en muros de bloques huecos de concreto

\begin{tabular}{crccc}
\hline Muro & $V_{m} / V_{\text {exp }}$ & $V_{C A L} / V_{\text {exp }}$ & $V_{N T C M} / V_{\text {exp }}$ & $V_{\text {VEN }} / V_{\text {exp }}$ \\
\hline $1(1)$ & 0.83 & 0.93 & 0.62 & 0.91 \\
$2(2)$ & 0.81 & 0.93 & 0.55 & 0.91 \\
$3(3)$ & 0.83 & 0.98 & 0.48 & 0.95 \\
$4(4)$ & 0.78 & 0.85 & 0.53 & 0.82 \\
$5(5)$ & 1.50 & 1.70 & 0.90 & 1.70 \\
$6(6)$ & 0.85 & 0.93 & 0.55 & 0.90 \\
$7(7)$ & 0.77 & 0.90 & 0.64 & 0.94 \\
$8(8)$ & 0.71 & 0.88 & 0.42 & 0.85 \\
$9(9)$ & 0.80 & 0.96 & 0.58 & 0.96 \\
$10(421)$ & 0.95 & 1.08 & 0.69 & 1.82 \\
$11(422)$ & 0.93 & 1.06 & 0.68 & 1.78 \\
$12(423)$ & 0.77 & 0.88 & 0.56 & 1.48 \\
$13(424)$ & 0.84 & 0.96 & 0.61 & 1.61 \\
$14(601)$ & 0.90 & 0.98 & 0.65 & 1.72 \\
$15(602)$ & 0.88 & 0.96 & 0.64 & 1.68 \\
$16(603)$ & 0.87 & 0.95 & 0.63 & 1.67 \\
$17(604)$ & 0.92 & 1.01 & 0.67 & 1.76 \\
Media & $\mathbf{0 . 8 8}$ & $\mathbf{1 . 0 0}$ & $\mathbf{0 . 6 1}$ & $\mathbf{1 . 3 2}$ \\
D estándar & 0.18 & 0.20 & 0.10 & 0.41 \\
C variación & 0.21 & 0.20 & 0.16 & 0.31 \\
\hline
\end{tabular}

Tabla 8. Valores de $\mathrm{F}_{\mathrm{v}}$ en la ecuación 19 Características de muros

Elaborados con bloques huecos de concreto 1(1) - 17(604)

Elaborados con tabicón jal-cemento 18(MD1) - 20(MD3) $F_{v}$

Elaborados con tabique rojo recocido 21(M2) - 25(804) 
Tabla 9. Aplicación en muros con varios castillos

\begin{tabular}{cccccc}
\hline Modelo & $\begin{array}{c}V_{m} . \\
\text { (ton) }\end{array}$ & $\begin{array}{c}V_{\text {castillos. }} \\
\text { (ton) }\end{array}$ & $\begin{array}{c}V_{\text {CAL }} \\
\text { (ton). }\end{array}$ & $\begin{array}{c}V_{\text {exp }} \\
\text { (ton) }\end{array}$ & $V_{\text {CAL }} / V_{\text {exp }}$ \\
\hline $\begin{array}{c}\text { 3D. planta } \\
\text { baja }\end{array}$ & 39.2 & 7.2 & 46.4 & 40.6 & 1.14 \\
\hline
\end{tabular}

Un punto importante del método es limitar el valor de la resistencia de la mampostería, $V_{m}$, que es función de las dimensiones del muro, propiedades mecánicas de la mampostería y castillos y del esfuerzo vertical. Ganz y Thurlimann (1985) y Woodward y Rankin (1985) realizaron ensayes en muros con relación $H / L$ igual a: 1.33 . 1.00. 0.67. 0.55 y 0.25 de los cuales concluyeron que la resistencia lateral permanece constante para niveles de $\sigma / f_{m}^{*}$ mayores a 0.25 donde $f_{m}^{*}$ representa la resistencia media a compresión de la mampostería. En los muros estudiados el valor máximo de $v_{u} / f_{m}^{*}=0.19$ ocurre en el muro 21(M2) y corresponde a una relación $\sigma / f_{m}^{*}=0.18$ y menor al límite experimental obtenido, como se muestra en la figura 9.

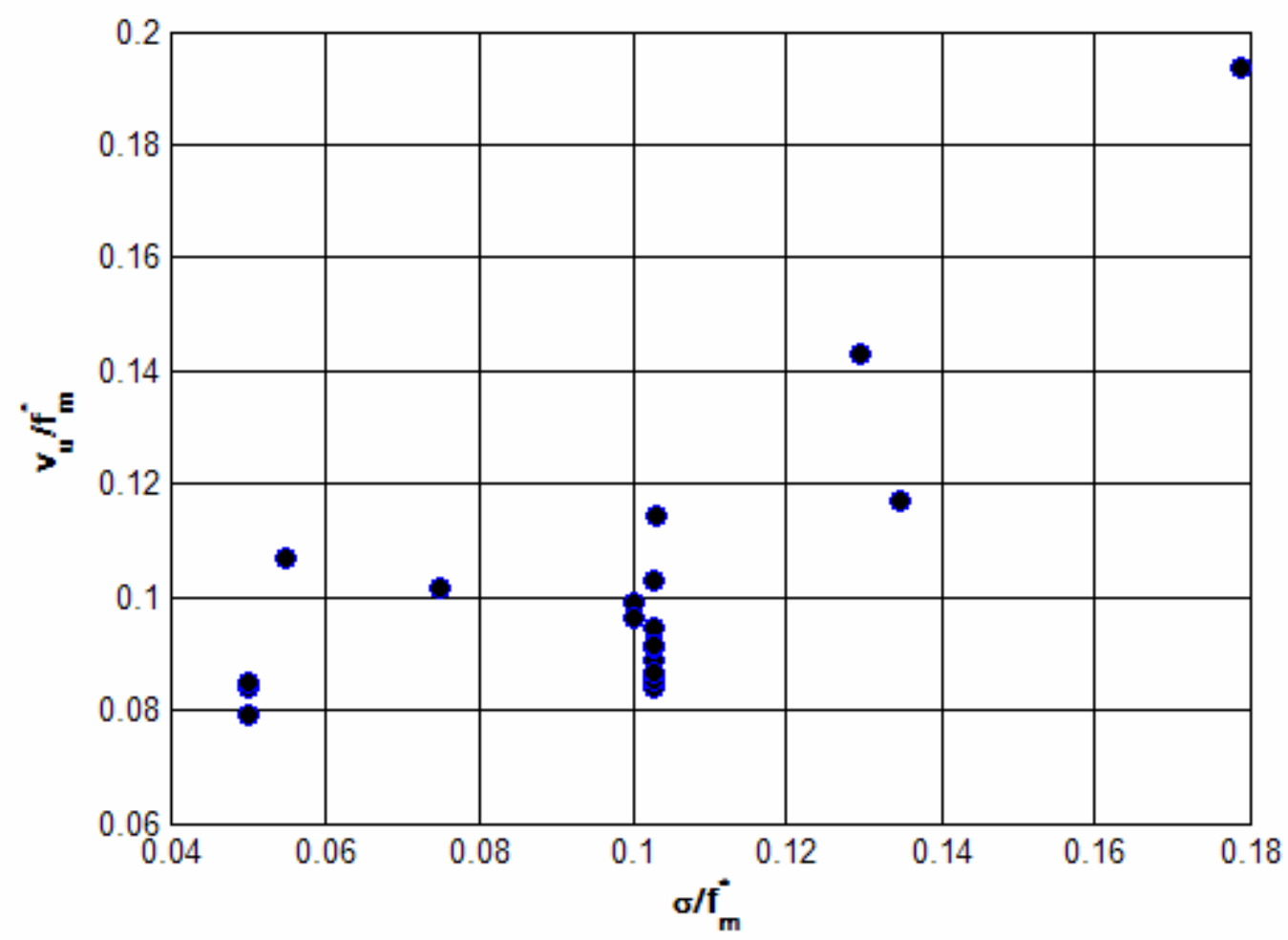

Figura 9. Influencia del esfuerzo vertical en la resistencia a cortante

\section{CONCLUSIONES}

El método semi-empírico aquí presentado fue aplicado a muros con relación de forma $H / L$ entre 0.74 y 1.26 con resultados aceptables e independientes del tipo de carga aplicada, monótona o cíclica, y del tipo de piezas utilizadas en la construcción. Para evaluar la resistencia de los muros se calculó la resistencia de la mampostería, de la sección de concreto de los castillos y del esfuerzo vertical aplicado. El 
parámetro fundamental para la aplicación es la resistencia a compresión diagonal $v_{m}^{*}$ que puede ser calculada fácilmente en ensayes de muretes.

El método propuesto tiene su base en la hipótesis de que la diagonal falla cuando el esfuerzo cortante excede la resistencia a compresión diagonal $v_{m}^{*}$ y tiene la ventaja respecto al método de la diagonal en compresión de no requerir un ancho equivalente para su aplicación. De esta forma se puede obtener la resistencia última y compararla con la resistencia de diseño. Es interesante mencionar que fue posible obtener el porcentaje de esfuerzo vertical participante en la resistencia y compararlo con el presentado en la normatividad.

En los resultados se observa que el porcentaje de resistencia aportada por la mampostería es de 88\% en muros de bloques huecos y de $76 \%$ en muros con piezas macizas, en el caso de los castillos esta aportación se invierte y en muros de bloques de concreto es de $12 \%$ y $22 \%$ en muros de piezas macizas. Respecto al promedio general se tiene un $85 \%$ de resistencia aportada por la mampostería y $15 \%$ de los castillos.

Finalmente, los autores proponen obtener el cortante último resistente en muros de mampostería considerando la aportación de la mampostería y de los castillos y agregar la influencia del esfuerzo vertical que en las condiciones normales no excede el $25 \%$ de la resistencia a compresión de la mampostería.

\section{AGRADECIMIENTOS}

Los autores agradecen al Programa del Mejoramiento del Profesorado (PROMEP-SEP) el apoyo económico para desarrollar el presente trabajo.

\section{REFERENCIAS}

Abdou, L (2005), “Modélisation du comportement mécanique des murs en maçonnerie charges dans leur plan”. PhD Tesis, Université Marné La Vallée, France.

Aguilar, G y S Alcocer (1997), "Efecto del refuerzo horizontal en el comportamiento de muros de mampostería confinada ante cargas laterales”, Informe técnico, CENAPRED.

ASTM (2007), Standard Test Method for Diagonal Tension (Shear) in Masonry Assemblages (E 519-07).

Andreaus, U (1996), "Failure criteria for masonry panels under in-plane loading”, Journal of the Structural Division, Vol. 122, No. 1, pp. 37-46.

Castilla, E y A Marinilli (2003), "Experiencias recientes en mampostería confinada de bloques de concreto”, IMME, Vol. 41, No. 2-3, pp. 28-39.

MINDUR (1987), “Norma de Estructura de Concreto Armado para Edificaciones. Análisis y diseño” COVENIN MINDUR 1753.

Cruz, I y A Mebarki (2002), "Resistance of masonry wind braced walls. Simplified model and experimental validation”, International Masonry Journal, Vol. 15, pp 73-79.

Flores, L y S Alcocer (2001), “Estudio analítico de estructuras de mampostería confinada”. Informe técnico, CENAPRED. 
Ganz, H y B Thurlimann (1985), “Tests on masonry walls subjected to axial load and shear”, Institute fur Baustatik and Konstruktion.

Hernández, E y D Urzúa (2002), “Pruebas dinámicas de resistencia sísmica de muros de mampostería confinada construidos con materiales pumíticos”, XIII Congreso Nacional de Ingeniería Estructural, pp. 337-348.

Mebarki, A, Q Bui, R Ami, P Delmotte y S Sanchez (2009), “A simplified model to asses the bearing capacity of masonry walls: theoretical predictions and experimental validations”. Construction and Building Materials Vol. 23, No. 2, pp. 1109-111, en prensa.

Meli, R y G Salgado (1969), “Comportamiento de muros de mampostería sujetos a carga lateral” Informe Técnico 237, Instituto de Ingeniería de la UNAM.

NTCC (2004b), "Normas técnicas complementarias para diseño y construcción de estructuras de concreto", Gobierno del Distrito Federal.

NTCM (2004a), "Normas técnicas complementarias para diseño y construcción de estructuras de mampostería”, Gobierno del Distrito Federal.

Sánchez, S, A Mebarki, A Soto y R Arroyo (2007), "Propiedades mecánicas de muretes y pilas con refuerzo metálico”, XVI Congreso Nacional de Ingeniería Sísmica.

Sánchez, S y A Mebarki (2006), “A Simplified Model of Shear-Capacity in Confined Masonry Walls”, Proceedings of the Eighth International Conference on Computational Structures Technology, pp. 210-218.

Treviño, E, S Alcocer, L Flores, R Larrua, J Zarate y L Gallegos (2004), “Investigación experimental del comportamiento de muros de mampostería confinada de bloques de concreto sometidos a cargas laterales cíclicas reversibles reforzados con acero de grados 60 y 42”, XIV Congreso Nacional de Ingeniería Estructural.

Woodward, K y F Rankin (1985), "Influence of block and mortar strength on shear resistance of concrete block masonry walls”, Report No NBSIR 85-3143, National Bureau of Standards. 